\title{
Bradykinesia akinesia inco-ordination test (BRAIN TEST): an objective computerised assessment of upper limb motor function
}

\author{
G Giovannoni, J van Schalkwyk, V U Fritz, A J Lees
}

University

Department of Clinical Neurosciences, Royal

Free and University College Medical School, London, UK

G Giovannoni

Department of Anaesthetics

J van Schalkwyk

Department of Neurology, University of the Witwatersrand, Johannesburg, South Africa

V U Fritz

Department of Clinical Neurology, University College Hospitals, London

A J Lees

Correspondence to: Dr Gavin Giovannoni, University Department of Clinical Neurosciences, Royal Free and University College Medical School, Royal Free Campus, Rowland Hill Street, London NW3 2PF. Telephone 0044 171794 0500; fax 0044171 431 1577; email g.giovannoni@rfhsm.ac.uk

Received 29 January 1999 and in revised form 12 April 1999

Accepted 28 May 1999.

\begin{abstract}
Objectives-A simple and rapid computerised keyboard test, based on the alternating finger tapping test, has been developed to quantify upper limb motor function. The test generates several variables: (1) kinesia score: the number of keystrokes in 60 seconds; (2) akinesia time: cumulative time that keys are depressed; (3) dysmetria score: a weighted index calculated using the number of incorrectly hit keys corrected for speed; (4) incoordination score: a measure of rhythmicity which corresponds to the variance of the time interval between keystrokes.
\end{abstract}

Methods-The BRAIN TEST ${ }^{\circ}$ was assessed on 35 patients with idiopathic Parkinson's disease, 12 patients with cerebellar dysfunction, and 27 normal control subjects.

Results-The mean kinesia scores of patients with Parkinson's disease or cerebellar dysfunction were significantly slower than normal controls (Parkinson's disease $=107$ (SD 28) keys/min $v$ cerebellar dysfunction $=86 \pm$ (SD 28) $v$ normal controls $=182$ (SD 26), $p<0.001$ ) and correlated with the UPDRS $(r=-0.69, \mathrm{p}<0.001)$. The akinesia time is very insensitive and was only abnormal in patients with severe parkinsonism. The median dysmetria (cerebellar dysfunction=13.8 $v$ Parkinson's disease $=6.1 v$ normal controls $=4.2, p=0.002$ ) and inco-ordination scores (cerebellar dysfunction $=5.12 v$ Parkinson's disease $=0.84 v$ normal controls $=0.15, p=0.002$ ) were significantly higher in patients with cerebellar dysfunction, in whom the dysmetria score correlated with a cerebellar disease rating scale $(r=0.64, p=0.02)$.

Conclusion-The BRAIN TEST ${ }^{\odot}$ provides a simple, rapid, and objective assessment of upper limb motor function. It assesses speed, accuracy, and rhythmicity of upper limb movements regardless of their physiological basis. The results of the test correlate well with clinical rating scales in Parkinson's disease and cerebellar dysfunction. The BRAIN test will be useful in clinical studies. It can be downloaded from the Internet (www.anaesthetist.com/ software/brain.htm).

(F Neurol Neurosurg Psychiatry 1999;67:624-629)

Keywords: Parkinson's disease; cerebellar dysfunction; rating scales; tests; kinesias; incoordination; dysmetria
Idiopathic Parkinson's disease is a chronic neurodegenerative disease predominantly affecting elderly people. It is characterised clinically by a tetrad of signs that include bradykinesia/akinesia, tremor, rigidity, and loss of postural righting reflexes. Motor impairment due to Parkinson's disease and other causes of parkinsonism is currently rated using the motor division of the unified Parkinson's disease rating scale (UPDRS). ${ }^{1}$ The UPDRS is the current "gold standard" for assessing therapeutic efficacy in clinical trials on patients with Parkinson's disease. By design the UPDRS is not an objective test and is subject to within and between assessor variability. ${ }^{23} \mathrm{We}$ therefore developed an objective, simple, rapid, and easy to perform computerised software program to assess upper limb motor function (BRAIN TEST ${ }^{\circ}$ ). The test is based on the finger tapping test ${ }^{4}$ but has the added advantage of providing information on incoordination and dysmetria, clinical signs associated with cerebellar dysfunction. ${ }^{5}$ Patients with cerebellar dysfunction are also bradykinetic but its is usually easy to differentiate them from patients with parkinsonism on clinical examination. ${ }^{5}$ In addition, some multisystem disorders such as multisystem atrophy ${ }^{6}$ and the spinocerebellar ataxias have both parkinsonism and cerebellar dysfunction. ${ }^{7}$ It would therefore be helpful if an objective test designed to assess bradykinesia could also provide information on cerebellar dysfunction. The primary aim of this study was to see if bradykinesia as measured by the BRAIN TEST correlated with the UPDRS. A secondary aim was to assess the utility of the BRAIN TEST in quantifying cerebellar dysfunction.

\section{Methods}

BRAIN TEST

The BRAIN TEST is a software program which is based on the alternating finger tapping test. ${ }^{4}$ It uses a standard personal computer with the keyboard as the test device. The two targets are the "S" and ";" keys which are $15 \mathrm{~cm}$ apart on the 101/102 keyboard, as well as the keyboards on most notebook size computers. The target keys are marked with adhesive red paper dots $10 \mathrm{~mm}$ in diameter. Test subjects are seated comfortably in front of the keyboard at a height that allows their arms to be above the keyboard when their elbows are flexed at $90^{\circ}$. Using the index finger the subject has to alternatively strike the target keys for a period of 60 seconds. Before starting the test the subjects are told to perform the test as fast and as 
Table 1 BRAIN TEST variables

(1) Kinesia score (KS): the number of keystrokes in 60 seconds

(2) Akinesia time (AT): the cumulative time that the keys are depressed for longer than $17 \mathrm{~ms}$

(3) Inco-ordination score (IS): a measure of rhythmicity, which corresponds to the variance of the time interval between keystrokes

(4) Dysmetria score (DS): a weighted index calculated using the number of incorrectly hit keys corrected for speed

accurately as possible. The data generated from this simple keyboard task are analysed to produce several variables (table 1 ). It takes less than 5 minutes to instruct a subject and to perform the test on both upper limbs. Version 1.0 of the BRAIN TEST can be downloaded free via the Institute of Neurology's website (www.ion.ucl.ac.uk).

The kinesia score (KS) is simply the total number of alternating keystrokes in $60 \mathrm{sec}-$ onds. The akinesia time (AT) is the cumulative time over the test period that any key is depressed for longer than $17 \mathrm{~ms}$. The incoordination score (IS) is a measure of rhythmicity, which corresponds to the variance of the time intervals between keystrokes. The less rhythmical the movement, the more variable the time between keystrokes becomes, the higher the IS. The dysmetria score (DS) is a weighted index calculated using the number of incorrectly hit keys corrected for speed. The missed keys are given a simple weighting based on their distance from the target key. The weighting uses a simple bulls eye target approach-that is, the keys immediately surrounding the target key are given the lowest weighting of 1 , the keys surrounding these an intermediate weighting of 2 , and any keys beyond these are given the highest weighting of 3 . This weighted score (from incorrectly hit keystrokes) is then

Table 2 Cerebellar disease rating scale (CDRS)

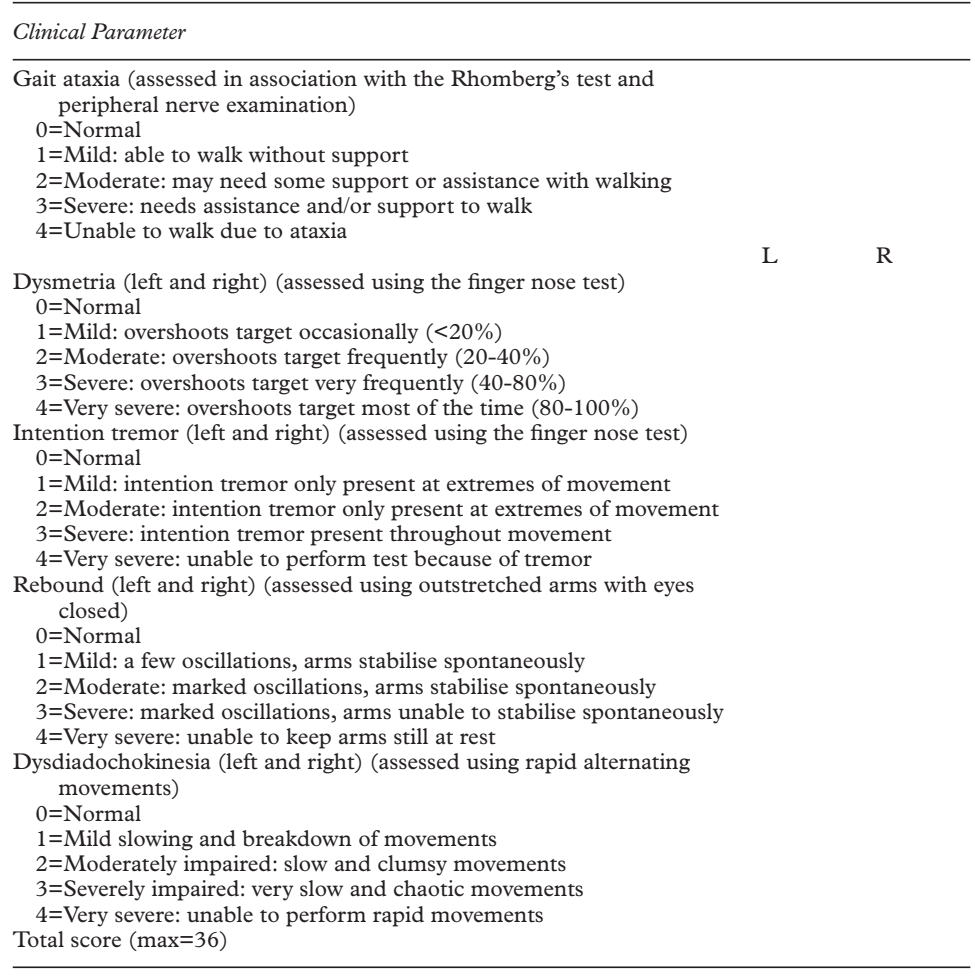

corrected for speed by dividing it by the KS (see Results: normal physiology section).

SUBJECTS

The BRAIN TEST was performed on 27 normal control subjects, 35 patients with Parkinson's disease, and 12 patients with predominantly cerebellar dysfunction. All the patients were recruited from the Neurology Department of the Johannesburg Hospital, South Africa. The normal controls were volunteers recruited from the general public. They were only included in the study if they had no underlying medical or psychiatric problems. Informed consent was obtained from all participating subjects. All subjects were assessed using the motor examination (items 18 to 31) of the UPDRS. ${ }^{1}$ Motor impairment due to cerebellar dysfunction was assessed with a simple cerebellar disease rating scale (CDRS, table 2), formulated for this study. This scale is currently being validated to see if it reliably measures neurological impairment and to see if it correlates with established disability and handicap scales. This will form the basis of a separate publication. To eliminate interrater reliability the same neurologist assessed all the patients. Hand preference was simply classified as dominant or non-dominant based on writing preference only.

\section{STATISTICAL ANALYSIS}

Normally distributed continuous variables were compared using a one way analysis of variance (ANOVA). If the ANOVA was significant individual groups were compared using a post hoc Tukey's honestly significant difference test (SPSS for Windows, release 7.5.1). Levene's test of homogeneity of variances was used to test if variables were normally distributed (SPSS for Windows, release 7.5.1). The distributions of all the variables, except the AT, were either normally distributed or could be normalised using a simple natural logarithmic transformation. ATs were compared using a Kruskal-Wallis one way analysis of variance. Non-parametric data were compared using Yates' corrected $\chi^{2}$ test and, if an expected value was less than 5 , the Fisher exact test. Continuous data were first normalised using a log transformation, and correlated using simple linear regression and a two tailed Pearson's test. A p value $<0.05$ was considered statistically significant.

\section{Results}

NORMAL PHYSIOLOGY

In normal controls the $\mathrm{KS}$ of the dominant hand was significantly higher than the nondominant hand (182 (SD 36) keystrokes/min $v$ 167 (SD 41) keystrokes/min, $\mathrm{p}=0.02$, paired $t$ test). The dominant hand was only quicker than the non-dominant hand in 21 out 35 normal control subjects $(60 \%)$. In the normal controls there was a negative correlation between the KS and age ( $n=35$, Pearson's correlation coefficient $=-0.38, \mathrm{p}=0.02)$. There were no significant differences between male and female normal control subjects. 

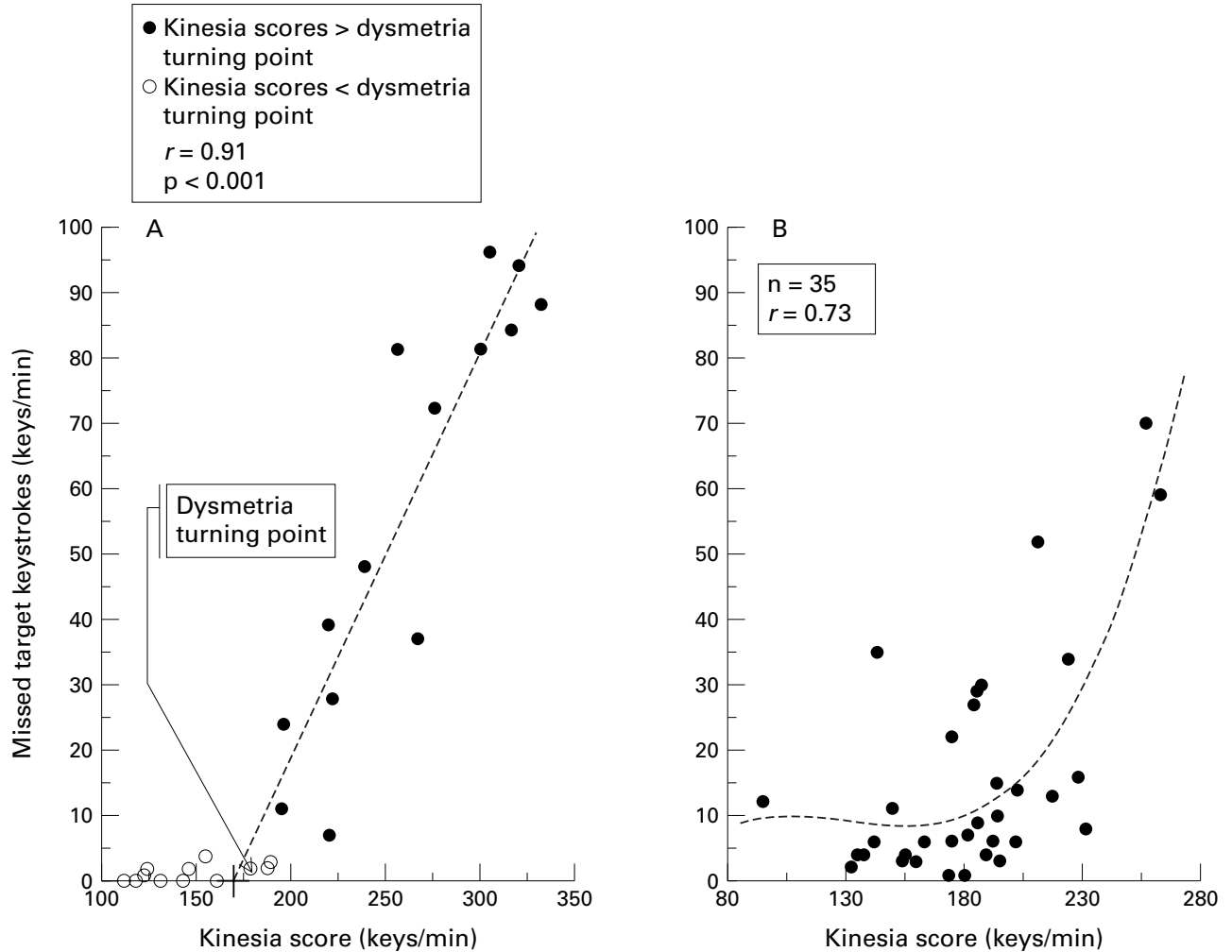

Figure 1 Normal physiology. Scatter plots of missed target keys $v$ the kinesia score from multiple tests performed by (A) a 28 year old normal man and (B) 35 normal control subjects showing the relation between the performance speed of the BRAIN TEST and the number of missed target key strikes. The plot shows that as the performance speed of the test increases the number of missed target strikes increases. The critical level at which this occurs is referred to for descriptive purposes as the dysmetria turning point. The dotted lines represent $(A)$ the linear, and $(B)$ polynomial regression derived from shaded points.

There was a non-linear relation between the with greater than 15 missed target keystrokes $\mathrm{KS}$ and incorrectly hit target keystrokes (fig $1 \mathrm{~A}$ during the 60 seconds of the test was and $\mathrm{B})$. This was due to subjects attempting to perform the test very quickly, which compromises accuracy and results in missed target keystrokes. The KS of the 10 normal controls significantly higher than 25 normal controls with less than 15 missed keystrokes (206 (SD 38) keystrokes/minute $v 172$ (SD 30) keystrokes/minute, $\mathrm{p}<0.01)$. The high inaccu-

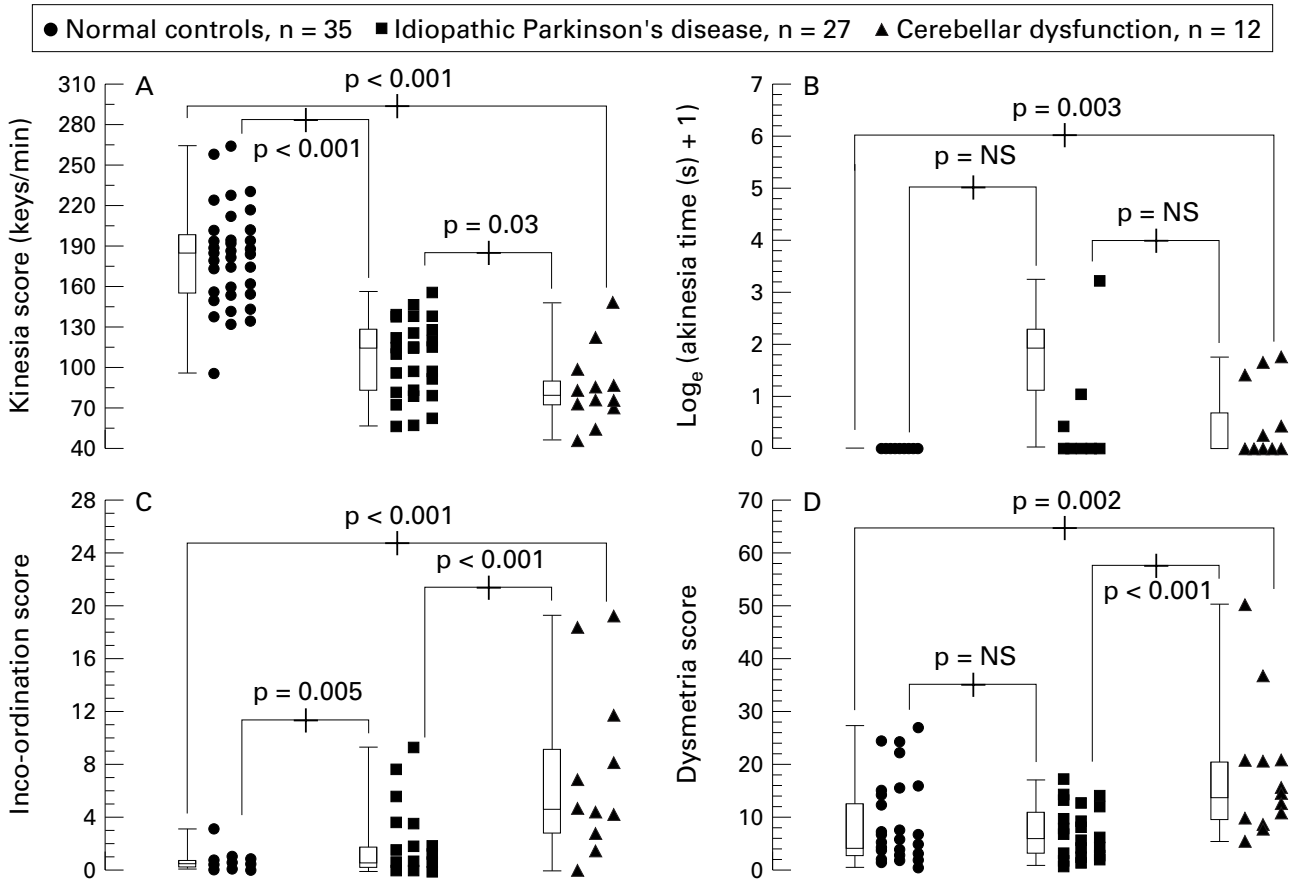

Figure 2 BRAIN TEST results. Scatter and box and whisker plots of the (A) kinesia score, (B) akinesia time, $(C)$ inco-ordination score, and (D) dysmetria score. The $p$ values were derived from a post hoc analysis of an ANOVA. 


\begin{tabular}{|c|c|c|c|c|}
\hline & Normal controls & $\begin{array}{l}\text { Idiopathic } \\
\text { Parkinson's Disease }\end{array}$ & Cerebellar disease & $p$ Value \\
\hline No of cases & 35 & 27 & 12 & \\
\hline Mean (SD) age (y) & $66(15)$ & $71(11)$ & $42(15)$ & $<0.001$ \\
\hline $\operatorname{Sex}(M: F)$ & $10: 25$ & 13:14 & $8: 4$ & NS \\
\hline Mean (SD) kinesia score (keys/min) & $182(36)$ & $107(28)$ & $86(28)$ & $<0.001$ \\
\hline Mean (range) akinesia time (s/min) & $0.0(0.0-0.00)$ & $0.99(0.00-4.8)$ & $1.07(0.00-24.4)$ & 0.003 \\
\hline Median inco-ordination ${ }^{\star}$ score (25th-75th \%tile) & $0.54(0.31-0.82)$ & $0.84(0.44-2.22)$ & $5.12(3.36-10.55)$ & 0.002 \\
\hline Median dysmetria* score (25th-75th \%tile) & $4.2(2.6-12.6)$ & $6.1(3.2-12.2)$ & $13.9(9.4-20.9)$ & 0.003 \\
\hline \multicolumn{5}{|l|}{ Post hoc Tukey's honestly significant difference test } \\
\hline Age & Kinesia score & Akinesia time & Inco-ordination score & Dysmetria score \\
\hline 一 & $\mathrm{PD}<\mathrm{NC}, \mathrm{p}<0.001$ & - & $\mathrm{PD}>\mathrm{NC}, \mathrm{p}=0.005$ & - \\
\hline $\mathrm{CB}<\mathrm{NC}, \mathrm{p}<0.001$ & $\mathrm{CB}<\mathrm{NC}, \mathrm{p}<0.001$ & $\mathrm{CB}<\mathrm{NC}, \mathrm{p}=0.003$ & $\mathrm{CB}>\mathrm{NC}, \mathrm{p}<0.001$ & $\mathrm{CB}>\mathrm{NC}, \mathrm{p}=0.002$ \\
\hline $\mathrm{CB}<\mathrm{PD}, \mathrm{p}<0.001$ & $\mathrm{CB}<\mathrm{PD}, \mathrm{p}<0.03$ & - & $\mathrm{CB}>\mathrm{PD}, \mathrm{p}<0.001$ & $\mathrm{CB}>\mathrm{PD}, \mathrm{p}<0.001$ \\
\hline
\end{tabular}

^Normalised using a $\log _{\mathrm{e}}$ transformation. $\mathrm{PD}=$ Parkinson's disease; $\mathrm{CB}=$ cerebellar dysfunction; $\mathrm{NC}=$ normal controls.

racy rate in these subjects does not mean that they have cerebellar dysfunction, but have simply performed the test too fast to ensure accuracy. This is clearly seen in fig $2 \mathrm{~A}$, in which a 28 year old male subject performed the test at increasing speeds. As the KS increased above 180 keystrokes/minute so did the frequency of missed target keystrokes. The KS above which inaccuracies creep in is referred to, for descriptive purposes, as the dysmetria turning point. In an attempt to compensate for this effect the weighted "bulls eye" score (from incorrectly hit keystrokes) was corrected for speed by dividing it by the KS.

\section{GROUP COMPARISONS}

Patients with cerebellar dysfunction had the following diagnoses; acute anticonvulsant toxicity (two), alcoholic cerebellar degeneration (two), subacute mercury poisoning (two), cerebellar onset multisystem atrophy (two), spinocerebellar ataxia (two), cerebellar stroke (two), chronic phenytoin induced cerebellar degeneration (one). Basic demographic and BRAIN TEST results are presented in table 3. Patients with cerebellar disfunction were significantly younger than both normal controls and patients with Parkinson's disease $(p<0.001)$.

For purposes of simplicity and brevity only the BRAIN TEST results from the dominant hand are presented. Analysis of the results from the non-dominant hand gave similar results. Patients with Parkinson's disease had significantly lower KS $(p<0.001)$ and higher IS $(p=0.005)$ than normal controls. Patients with cerebellar dysfunction had significantly lower KS $(\mathrm{p}<0.001)$ and higher AT $(\mathrm{p}=0.003)$, IS $(\mathrm{p}<0.001)$, and DS $(\mathrm{p}=0.002)$ than normal controls. Patients with cerebellar dysfunction also had significantly lower KS $(p=0.03)$ and higher IS $(\mathrm{p}<0.001)$ and DS $(\mathrm{p}<0.001)$ than patients with Parkinson's disease.

CORRELATIONS WITH DISEASE RATING SCALES In patients with Parkinson's disease the UPDRS (18-31) correlated significantly with the KS $(r=-0.69, \mathrm{p}=0.001$, fig $3 \mathrm{~A})$ and the IS $(r=0.41, \mathrm{p}=0.02)$ and tended to correlate with the AT $(r=0.35, p=0.07)$. The UPDRS (1831) did not correlate with the DS $(r=0.26$, $\mathrm{p}=0.18)$. Similar correlations were found when using the total UPDRS (data not shown). In patients with cerebellar dysfunction the
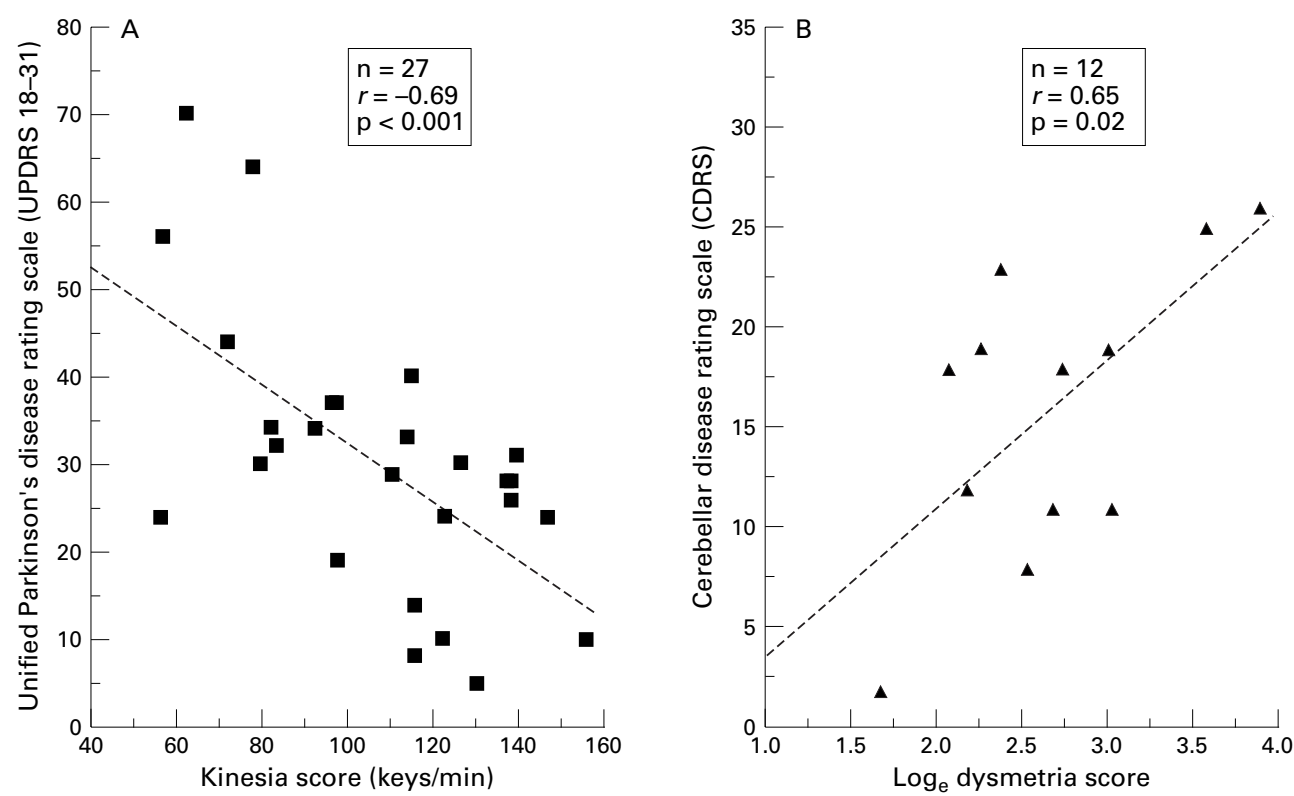

Figure 3 Disease rating scales. (A) UPDRS v kinesia score: scatter plot of the motor part, items 18 to 31, of the unified Parkinson's disease rating scale (UPDRS) ${ }^{1} v$ the kinesia score in 27 patients with idiopathic Parkinson's disease. (B) $C D R S v$ the natural logarithm of the dysmetria score: scatter plot of the cerebellar disease rating scale (CDRS, table 2) $v$ the dysmetria score (DS) in 12 patients with cerebellar dysfunction. The dotted lines represent simple linear regressions. 
CBDRS correlated significantly with the KS $(r=-0.38, \mathrm{p}=0.03)$ and DS $(r=0.63, \mathrm{p}=0.03$, fig $3 \mathrm{~B}$ ) and tended to correlate with AT $(r=-0.55, \mathrm{p}=0.06)$. The CBDRS did not correlate with the IS $(r=0.3, \mathrm{p}=0.3)$.

\section{Discussion}

Akinesia (defined as a delay in initiating a movement or switching between two defined movements) and bradykinesia (defined as slowness of movement) are the primary abnormalities in motor function in patients with parkinsonism. ${ }^{8}$ They are not unique to parkinsonism and occur in patients with frontal lobe disease $^{9}$ and cerebellar dysfunction. ${ }^{5}$ In patients with Parkinson's disease these cardinal signs usually respond to dopamine replacement therapy. Currently this response is monitored clinically with various rating scales and timed tasks. ${ }^{10}$ Objective ancillary tests such as the finger tapping ${ }^{4}$ and peg in hole ${ }^{11}$ tests provide quantitative data but are limited to measuring bradykinesia alone. In addition to measuring bradykinesia the BRAIN TEST has an advantage over these other tests by providing additional information on the nature of the motor dysfunction.

The KS is a simple measure of speed of movement and is hence a surrogate for bradykinesia. Patients with Parkinson's disease and cerebellar dysfunction were, as expected, slower than normal. Patients with cerebellar dysfunction were significantly younger than normal controls and patients with Parkinson's disease. This would if anything result in an underestimation of the observed differences in KS, which was significantly lower in the patients with cerebellar dysfunction. In principle the AT should provide information on the delay in initiating or switching between movements. Unfortunately the utility of the AT is limited by its lack of sensitivity, which is dictated by the repeat rate of the keyboard and computer operating system. However, in patients with severe parkinsonism and cerebellar dysfunction the AT is clearly prolonged and may prove useful as an objective measure of akinesia. With future hardware and software developments the sensitivity of the AT will improve. The IS and DS are the most sensitive to cerebellar dysfunction, providing information on the loss of rhythmicity of repetitive movements or dysdiadochokinesia and dysmetria, respectively.

The term arrhythmokinesis, coined by Wertham ${ }^{12}$ to describe the disturbance in rapid alternating ballistic movements in patients with cerebellar dysfunction, probably best describes the abnormality measured by the IS. Arrhythmokinesis has also been described in Parkinson's disease albeit to a lesser degree, ${ }^{13-15}$ which supports our findings of an increased IS in patients with Parkinson's disease, but not to the same degree as patients with cerebellar dysfunction. This would also be in keeping with the clinical signs of these two motor syndromes. The principle of the IS - that is, the variance of the time between keystrokes-has previously been used in a simple, but not alternating, computerised tapping test. ${ }^{16}$ The inves- tigators who developed this test found that the SD of the time between keystrokes was significantly higher in patients with cerebellar disease than patients with parkinsonism. The differences between the groups were however small, possibly due to the design of the test, which only lasts 15 seconds and does not require alternating movements across a distance. Modifying the test by introducing alternating movements over a longer time period has amplified these differences. Importantly the $\mathrm{KS}$ and DS correlate with clinical severity as rated using the UPDRS and CDRS, making them useful surrogate makers by which to assess clinical impairment. Unfortunately the CDRS has not been fully validated as a clinical scale. However, as it is based on the scoring system used in the UPDRS, it should provide a reasonable assessment of neurological impairment due to cerebellar dysfunction.

Although the patients with cerebellar dysfunction were heterogeneous, with some patients having multisystem diseases, they were selected because of a predominance of cerebellar signs. This allowed us to assess the influence of cerebellar dysfunction on the performance of the test. The fact that the patients with cerebellar dysfunction were more bradykinetic than the patients with Parkinson's disease in this study is relatively unimportant, as the level of bradykinesia depends on severity of disease. This is difficult to control for when the impairment and disability due to dysfunction of these two motor systems is so disparate. The KS (bradykinesia), AT (akinesia), and IS (arrhythmokinesis) cannot be used to distinguish between cerebellar dysfunction and parkinsonism. However, the DS (dysmetria) seems to be selectively increased in cerebellar dysfunction. This may be useful as a screening test in patients with parkinsonism for concomitant cerebellar disease. We must emphasise that the BRAIN TEST is not a substitute for a clinical examination, in assessing the motor system.

Our results confirm findings by others that the dominant hand is faster than the nondominant hand. ${ }^{16}$ However, other confounding factors that have not been considered in this study include age, sex, educational level, cognitive functioning, mood, practice effects, and subclinical disease, all of which have the potential to affect the test. The effect of these factors has been considered in a large study using the BRAIN TEST, which demonstrates an association with sex; males have higher KS compared with females, confirming previous findings ${ }^{12}$ and an association between educational level and the performance of the BRAIN TEST (unpublished data). ${ }^{17}$

The primary aim of the BRAIN TEST is not to separate out patients with parkinsonism from those with cerebellar dysfunction. It provides object scores of upper limb motor function, which include aspects of speed, accuracy, and rhythmicity regardless of their physiological basis. Other disorders of motor function including spasticity, weakness, tremor, dystonia, chorea, athetosis, and apraxia also affect the performance of this test. The utility of the BRAIN TEST as an objective 
measure of motor dysfunction in these disorders needs to be investigated.

In conclusion, the BRAIN TEST is a simple, rapid, and objective test, which is designed to assess upper limb motor function. It is a significant improvement on the finger tapping test as it provides additional qualitative information on the accuracy and rhythmicity of upper limb movement. In patients with Parkinson's disease or cerebellar dysfunction the kinesia and dysmetria scores correlate with clinical disease rating scales. The BRAIN TEST is proving useful as a tool for objectively quantifying upper limb motor function in clinical practice and would be particularly useful in clinical studies. It would also be useful in longitudinal studies as it provides a qualitative, objective, index. Longitudinal decrease in the KS may be a sensitive index for early detection of Parkinson's disease. The ubiquitous availability of personal computers and distribution of the BRAIN TEST via the Internet should make it easy to implement on a wider scale.

We thank the Bobby Grieve Research Fund, University of the Witwatersrand, Johannesburg, South Africa, for a generous grant that made this study possible.

1 Fahn S, Elton RL, members of the UPDRS committee. Unified Parkinson's disease rating scale. In: Fahn S, Marsden CD, Goldstein M, et al, eds. Recent developments in Parkinson's disease. 2nd ed. New York: Macmillan, 1987:153-63.

2 Henderson L, Kennard C, Crawford TJ, et al. Scales for rating motor impairment in Parkinson's disease: studies of reliability and convergent validity. $\mathcal{f}$ Neurol Neurosurg Psychiatry 1991;54:18-24.

3 Martinez-Martin P, Gilnagel A, Gracia LM, et al. Unified Parkinson's disease rating scale characteristics and structure. Mov Disord 1994;9:76-83.

4 Burns BD, De Jong JD. A preliminary report on the measurement of Parkinson's disease. Neurology 1960;10: 1096-102.

5 Diener H-C, Dichgans J. Pathophysiology of cerebellar ataxia. Mov Disord 1992;7:95-109.

6 Wenning GK, Kraft E, Beck R, et al. Cerebellar presentation of multiple system atrophy. Mov Disord 1997;12:115-17.

7 Sasaki H, Fukazawa T, Yanagihara T, et al. Clinical features and natural history of spinocerebellar ataxia type 1. Acta Neurol Scand 1996;93:64-71.

8 Marsden CD. The mysterious motor function of the basal ganglia: the Robert Wartenburg lecture. Neurology 1982;32: 514-39.

9 Watson RT, Miller BD, Heilman KM. Nonsensory neglect. Ann Neurol 1978;3:505-8.

10 Lakke JPWF. Assessment and measurement. In: Stern GM, ed. Parkinson's disease. London: Chapman and Hall Medical, 1990:467-91.

11 Godwin-Austen RB, Tomlinson EB, Frears CC, et al. Effects of L-dopa in Parkinson's disease. Lancet 1969;ii: $165-8$.

12 Wertham FI. A new sign of cerebellar diseases. 7 Nerv Ment Dis 1929;69:486-93.

13 Nakamura R, Nagasaki H, Narabayashi H. Disturbances of rhythm formation in patients with Parkinson's disease, I: characteristics of tapping responses to periodic signals. Percept Mot Skills 1987;46:63-75.

14 Nagasaki H, Narabayashi $H$, Taniguchi R. Disturbances of rhythm formation in patients with Parkinson's disease, II: a forced oscillation model. Percept Mot Skills 1987;46:79-87.

15 Sheridan MR, Flowers KA. Movement variability and bradykinesia in Parkinson's disease. Brain 1990;113:114961.

16 Shimoyama I, Ninchoji T, Uemura K. The finger-tapping test. A quantitative analysis. Arch Neurol 1990;47:681-4.

17 Homann CN, Suppan K, Wenzel K, et al. The brain test, a portable, fast easy and objective means to evaluate patients with Parkinson's disease. Mov Disord 1998;13(supplement 2):211. 